\title{
A Novel Ferroptosis-Related Long Non-coding RNA Prognostic Risk Model for Gastric Cancer
}

\section{Shenglan Huang}

Nanchang University Second Affiliated Hospital

Dan Li

Nanchang University Second Affiliated Hospital

\section{Lingling Zhuang}

Nanchang University Second Affiliated Hospital

Liying Sun

Nanchang University Second Affiliated Hospital

Jianbing Wu ( $\nabla$ hhgwjb@163.com )

Nanchang University Second Affiliated Hospital https://orcid.org/0000-0002-4576-7286

\section{Research}

Keywords: gastric cancer, IncRNA, ferroptosis-related, prognostic model, nomogram

Posted Date: July 21st, 2021

DOI: https://doi.org/10.21203/rs.3.rs-718468/v1

License: (c) (1) This work is licensed under a Creative Commons Attribution 4.0 International License. Read Full License 


\section{Abstract}

Background囚Gastric cancer (GC) is one of the most common malignant tumors with a poor prognosis. Ferroptosis is a novel and distinct type of non-apoptotic cell death that is closely associated with metabolism, redox biology, and tumor prognosis. Recently, ferroptosis-related long non-coding RNAs (IncRNAs) have received increasing attention in predicting cancer prognosis. Thus, we aimed to construct an ferroptosis-related IncRNAs signature for predicting the prognosis of patients with gastric cancer.

Methods $\triangle$ We built an ferroptosis-related IncRNA risk signature by using Cox regression based on TCGA database. Kaplan-Meier survival analysis was conducted to compare the overall survival (OS) in different risk groups. Cox regression was performed to explore whether the signature could be used as an independent factor. A nomogram was built involving the risk score and clinicopathological features. Furthermore, we explored the biological functions and immune states in two groups.

Results冈Eight ferroptosis-related IncRNAs were obtained for constructing the prognosis model in gastric cancer. Kaplan-Meier curve analysis revealed that patients in the high-risk group had worse survival than those in the low-risk group. The survival outcome was also appropriate for subgroup analysis, including age, sex, grade, and clinical stage. Multivariate Cox regression analysis and receiver operating characteristic (ROC) curve analysis demonstrated that the risk score was an independent prognostic factor and superior to traditional clinicopathological features in predicting GC prognosis. Next, we established a nomogram according to clinical parameters (age, sex, grade, and clinical stage) and risk score. All the verified results, including ROC curve analysis, calibration curve, and decision curve analysis, demonstrated that the nomogram could accurately predict the survival of patients with gastric cancer. Gene set enrichment analysis revealed that these IncRNAs were mainly involved in cell adhesion, cancer pathways, and immune function regulation.

Conclusion: We established a novel ferroptosis-related prognostic risk signature including eight IncRNAs and constructed a nomogram to predict the prognosis of gastric cancer patients, which may improve prognostic predictive accuracy and guide individualized treatment for patients with GC.

\section{Background}

Gastric cancer (GC) is the second leading cause of cancer-related mortality and ranks fifth in terms of incidence rates worldwide $(1,2)$. Although systemic chemotherapy, surgery, radiotherapy, targeted therapy, and immunotherapy have proven efficacy against GC, the prognosis of GC remains poor. According to statistics, the annual fatality rate of GC is approximately $10 \%$, and the 5 -year survival rate is less than $30 \%$ (3). Such poor survival is mainly because of the low early diagnosis rate of GC, rapid progression, chemotherapy resistance, high heterogeneity in patients with GC, and a lack of early prognostic indicators (4). Currently, tumor size, lymph node metastasis, distant metastasis, stage, and pathological type of cancer are the main factors for prognosis (5). However, the high heterogeneity in patients with GC may affect the accuracy and applicability of the current prediction methods. Therefore, it is imperative to 
explore highly sensitive and specific molecular markers to improve the predictive accuracy and prognostic specificity in patients with GC.

Ferroptosis, a novel and distinct type of non-apoptotic cell death, is characterized by the accumulation of iron-dependent reactive oxygen species (ROS), oxidization of polyunsaturated fatty acids (PUFAs), accumulation of lipid peroxides, and phospholipid peroxidation damage of cell membranes $(6,7)$. It is markedly distinct from other forms of regulated cell death, including apoptosis, autophagy, and necroptosis. Studies have verified that ferroptosis is closely associated with numerous biological processes, such as lipid metabolism, amino acid metabolism, iron metabolism, and glutathione metabolism, as well as with key regulators, including GPX4, NRF2, FSP1, and p53 (8). Recently, numerous studies have evaluated the important role of ferroptosis in tumor prognosis (9). A ferroptosis-related gene prognostic model was successfully constructed for GC (10). Nonetheless, mRNA signatures may provide imperfect predictions because of low tissue specificity and instability (11). Hence, it is vital to discover novel ferroptosis-related biomarkers for GC prognosis.

Long non-coding RNAs (IncRNAs), which account for $80-90 \%$ of all ncRNAs and are structurally more than 200 nucleotides (nt) in length, have attracted widespread attention as cancer biomarkers for early disease diagnosis and prognosis, as potential therapeutic targets, and in drug resistance $(4,12,13)$. Notably, IncRNAs also participate in ferroptosis and play a crucial role in regulating ferroptosis in cancer. Some IncRNAs can act as competitive endogenous RNAs to prevent oxidation, thereby inhibiting ferroptosis. It was reported that IncRNA LINC00336 level is upregulated in lung cancer and plays a role in inhibiting ferroptosis by interacting with ELAV-like RNA-binding protein(14). LINC00336 also serves as an endogenous sponge of microRNA6852 to regulate the expression of cystathionine- $\beta$-synthase (CBS), which is a surrogate marker of ferroptosis (14). Mao et al. (15) revealed that the p53-related IncRNA P53RRA promotes ferroptosis and suppresses cancer progression by directly interacting with the functional domain of the signal protein and activating the p53 pathway. In addition, ferroptosis-related IncRNAs were chosen as excellent indicators for predicting survival in head and neck squamous cell carcinoma (16). However, the potential value of ferroptosis-related IncRNAs as prognostic indicators and therapeutic targets has not been explored in patients with GC.

Therefore, in this study, we aimed to construct a ferroptosis-related IncRNA prognostic model and nomogram based on the Cancer Genome Atlas (TCGA) data and then explore the biological functions and related immune responses of ferroptosis-related IncRNAs in GC. A flowchart of this study is shown in Fig. 1.

\section{Results}

\section{Identification of Ferroptosis-related LncRNAs in GC}

First, the mRNA and IncRNA expression matrix and clinical information of $375 \mathrm{GC}$ samples were acquired from the TCGA database. After removing the data of repetitive samples, patients with incomplete clinical 
pathological data, and patients with less than 30 days follow-up time, the data of 334 patients with complete follow-up information and 296 patients with complete clinicopathological data were included in this study for subsequent analysis. The clinical pathological features of patients with GC included in this study are shown in Table 1. Then, the data of 259 ferroptosis-related genes were downloaded from FerrDb. The data included information of 217 ferroptosis-related genes obtained from the TCGA database using "limma" R package. According to the Pearson correlation coefficient analysis results, 741 ferroptosis-related IncRNAs were identified based on the filtering criteria of correlation coefficient $<0.3$ and $\mathrm{p}<0.001$. 
Table 1

Clinical pathological parameters of patients with gastric cancer based on The Cancer Genome Atlas database

\begin{tabular}{|c|c|c|c|}
\hline Characteristic & Primary cohort $(n=296)$ & Validation cohort $(n=146)$ & $p$ value \\
\hline Age (year) & & & 0.734 \\
\hline$<=65$ & 135 & 69 & \\
\hline$>65$ & 161 & 77 & \\
\hline Gender & & & 0.348 \\
\hline Male & 186 & 85 & \\
\hline Female & 110 & 61 & \\
\hline Tumor grade & & & 0.452 \\
\hline G1 & 7 & 1 & \\
\hline G2 & 101 & 52 & \\
\hline G3 & 188 & 93 & \\
\hline Clinical stage & & & 0.853 \\
\hline I & 38 & 15 & \\
\hline II & 97 & 49 & \\
\hline III & 130 & 68 & \\
\hline IV & 31 & 14 & \\
\hline T classification & & & 0.9 \\
\hline T1 & 13 & 7 & \\
\hline T2 & 61 & 26 & \\
\hline T3 & 145 & 72 & \\
\hline T4 & 77 & 41 & \\
\hline N classification & & & 0.826 \\
\hline NO & 90 & 40 & \\
\hline N1 & 80 & 43 & \\
\hline N2 & 64 & 29 & \\
\hline N3 & 62 & 34 & \\
\hline
\end{tabular}

Note: T, tumor size; $\mathrm{N}$, lymph node; $\mathrm{M}$, distant metastasis 


\begin{tabular}{|c|c|c|c|}
\hline Characteristic & Primary cohort $(n=296)$ & Validation cohort $(n=146)$ & $\mathrm{p}$ value \\
\hline M classification & & & 0.918 \\
\hline MO & 277 & 137 & \\
\hline M1 & 19 & 9 & \\
\hline \multicolumn{4}{|c|}{ Note: T, tumor size; $N$, lymph node; $M$, distant metastasis } \\
\hline
\end{tabular}

To obtain prognostic-associated ferroptosis-related IncRNAs, we first conducted univariate Cox regression analysis and log-rank tests according to $P<0.05$. Among the 741 ferroptosis-related IncRNAs, 16 were identified as prognostic-associated candidates that were significantly correlated with the survival of patients with GC, and the results are shown in Table 2. Subsequently, eight IncRNAs were further filtered to construct a prognostic signature using multivariate Cox regression analysis based on the minimum AIC value (AIC = 1213), which were AL365181.3, MIR3142HG, PVT1, LINC01315, AL353804.1, HAGLR, AC005586.1, and AC245041.1. Among them, AL365181.3, MIR3142HG, PVT1, LINC01315, AL353804.1, and AC005586.1 were prognostic IncRNAs with HR $<1$, and HAGLR and AC245041.1 were prognostic atrisk IncRNAs with $H R>1$, as shown in Fig. 2. Kaplan-Meier survival analysis intuitively displayed the relationship between the expression of eight ferroptosis-related IncRNAs and survival. The results showed that all IncRNAs were significantly correlated with survival ( $p<0.05)$, and AL365181.3, MIR3142HG, PVT1, LINC01315, AL353804.1, and AC005586.1 were positively correlated with survival, whereas the other two IncRNAs (HAGLR, AC245041.1) were negatively correlated with survival (Fig. 3A-H). According to the data of the prognostic IncRNAs obtained from the Cox regression analysis, we constructed a co-expression network of the eight ferroptosis-related IncRNA and mRNAs using Cytoscape and Sankey diagrams to visually present the correlation among ferroptosis-related genes, ferroptosis-related IncRNAs, and risk types of IncRNAs (Fig. 4A-B). 
Table 2

Information of 16 ferroptosis-related IncRNAs significantly correlated with the survival of patients with gastric cancer

\begin{tabular}{|llllllll|}
\hline InCRNA & KM & B & SE & HR & HR.95L & HR.95H & p value \\
\hline IP05P1 & 0.029 & -0.26 & 0.105 & 0.771 & 0.628 & 0.948 & 0.014 \\
\hline AC084033.3 & 0.008 & 0.177 & 0.089 & 1.194 & 1.002 & 1.423 & 0.048 \\
\hline AP003392.1 & 0.01 & -0.238 & 0.106 & 0.788 & 0.64 & 0.97 & 0.025 \\
\hline AL365181.3 & 0.046 & -0.04 & 0.019 & 0.96 & 0.924 & 0.998 & 0.037 \\
\hline MIR3142HG & 0.031 & -0.226 & 0.092 & 0.798 & 0.666 & 0.955 & 0.014 \\
\hline PVT1 & 0.003 & -0.15 & 0.053 & 0.861 & 0.775 & 0.955 & 0.005 \\
\hline LINC01315 & 0.024 & -0.239 & 0.088 & 0.787 & 0.663 & 0.936 & 0.007 \\
\hline ASMTL-AS1 & 0.016 & -0.164 & 0.077 & 0.848 & 0.729 & 0.988 & 0.034 \\
\hline LINC00106 & 0.002 & -0.176 & 0.071 & 0.838 & 0.729 & 0.964 & 0.014 \\
\hline AL353804.1 & 0.001 & -0.28 & 0.141 & 0.755 & 0.573 & 0.996 & 0.046 \\
\hline SREBF2-AS1 & 0.023 & -0.351 & 0.148 & 0.704 & 0.527 & 0.94 & 0.018 \\
\hline HAGLR & 0.001 & 0.092 & 0.032 & 1.096 & 1.033 & 1.163 & 0.002 \\
\hline AC245041.2 & 0.018 & 0.095 & 0.036 & 1.1 & 1.025 & 1.179 & 0.008 \\
\hline TNFRSF10A-AS1 & 0.0124 & -0.0966 & 0.048 & 0.909 & 0.827 & 0.998 & 0.046 \\
\hline AC005586.1 & 0.000 & -0.275 & 0.095 & 0.76 & 0.631 & 0.915 & 0.004 \\
\hline AC245041.1 & 0.036 & 0.032 & 0.008 & 1.032 & 1.015 & 1.049 & 0.000 \\
\hline Note: HR: hazard ratio; HR.95L: low $95 \%$ Cl of HR; HR.95H: high $95 \%$ Cl of HR. & \\
\hline
\end{tabular}

\section{Construction and Verification of Ferroptosis-Related LncRNA Prognostic Model for GC}

According to the eight prognostic ferroptosis-related IncRNAs and corresponding regression coefficients, the risk score of each patient was obtained according to the follow formula:

risk score $=(0.143 \times$ HAGLR expression $)+(-0.212 \times$ AC005586.1 expression $)+(-0.055 \times$ AL365181.3 expression $)+(-0.242 \times$ MIR3142HG expression $)+(-0.089 \times$ PVT1 expression $)+(-0.192 \times$ LINC01315 expression $)+(-0.409 \times$ AL353804.1 expression $)+(0.025 \times$ AC245041.1 expression $)$.

A ferroptosis-related IncRNA prognostic signature was established, and patients with GC were divided into two groups: low- and high-risk groups (median value $=1.1064$ ). Kaplan-Meier survival curves for OS 
demonstrated that patients with GC in the high-risk group had poorer survival than those in the low-risk group $(p=1.81 e-07)$, as shown in Fig. 5A. Time-ROC curve analysis was performed to evaluate the precision of the prognostic model; the AUC was calculated, and the results proved that the risk score model provided a precise predictive role. The AUC values for the 1-, 2-, and 3-year OS by the prognostic model were $0.728,0.755$, and 0.759 , respectively (Fig. $5 B$ ). Risk score curves and scatter plots were drawn to explain the relationship between the risk score and survival status in all included patients with $\mathrm{GC}$, and the results revealed that the higher the risk score, the higher was the mortality rate observed in patients with GC (Fig. 5C-D). The heatmap also suggested that six IncRNAs (AL365181.3, MIR3142HG, PVT1, LINC01315, AL353804.1, and AC005586.1) were upregulated in the low-risk group, whereas the other two IncRNAs (AC245041.1 and HAGLR) were upregulated in the high-risk group (Fig. 5E). In addition, we carried out verification analysis of the eight prognostic IncRNAs in the validation cohort and found that the survival probability in the high-risk group was significantly lower than that in the low-risk group $(p=$ $2.58 \mathrm{e}-06$ ), and the predictive validity of AUC values for the $1-, 2-$, and 3 -years were $0.760,0.740$, and 0.732 , respectively. The results of the risk score curves, scatter plots, and heatmap in the test group showed similar trends to those observed in the primary set (Fig. 6A-E). Subsequently, we adopted stratification analysis to estimate the relevance of risk scores and related clinicopathological parameters. The results showed that patients with GC in different subgroups had worse survival in the high-risk group, including age ( $\leq 65$ and $>65$ years), gender (male and female), grade (grade 1-2 and grade 3 ), and clinical stage (stage I-II and stage III-IV). This indicated that the prognostic signature was applicable to different subtypes of GC (Fig. 7A-H).

Furthermore, univariate and multivariate Cox regression analyses were performed to identify whether the risk score model was independently associated with the survival of patients with GC. Univariate Cox analysis showed that clinical characteristics of age $(\mathrm{HR}=1.022(1.003-1.042), 95 \% \mathrm{Cl}: 1.003-1.042, p$ $=0.024)$, clinical stage $(H R=1.478,95 \% \mathrm{Cl}: 1.172-1.863$, $p \otimes 0.001)$, Tumor $(T, H R=1.289,95 \% \mathrm{Cl}: 1.013-$ $1.641, p=0.039)$, Node $(\mathrm{N}, \mathrm{HR}=1.252(1.053-1.490), 95 \% \mathrm{Cl}: 1.053-1.590, p=0.011)$, and risk score $(\mathrm{HR}=1.097,95 \% \mathrm{Cl}: 1.063-1.132, p \otimes 0.001)$ were associated with OS (Fig. 8A). Multivariate Cox regression analysis demonstrated that age $(\mathrm{HR}=1.033,95 \% \mathrm{Cl}: 1.012-1.054, p=0.02)$ and risk score $(\mathrm{HR}=1.097,95 \% \mathrm{Cl}: 1.061-1.133, p<0.001)$ were independent survival prognosis factors for patients with GC (Fig. 8C). Multi-ROC curve analysis proved that the risk score model provided a more precise predictive role for survival than other clinical parameters (age, sex, grade, stage, $T, N, M$ ), with AUC $=0.738$ (Fig. 8E). Cox regression and multi-ROC curve analyses were performed for the validation group, as shown in Fig. 8 (B, D, F). The results indicated that the risk score was independently associated with survival in the validation group and that the risk score model was an excellent predictive indicator of prognosis than other clinical parameters with $A U C=0.771$. The above results demonstrated that the ferroptosis-related IncRNA prognostic model had an accurate predictive ability for prognosis in patients with GC.

\section{Construction and Evaluation of the Prognostic Nomogram}


A nomogram was established to predict the 1- and 3-year survival probability of patients with GC based on age, sex, grade, clinical stage, and risk score. The comprehensive score was calculated by combining each clinical factor, the highest total score, and the worst prognosis (Fig. 9A). The accuracy and consistency of the nomogram for 1- and 3-year OS were assessed using a calibration curve, multi-ROC curve, and DCA. The results of the calibration curve showed that the prognostic nomogram model was nearly in accordance with reality, as shown in Fig. 9B-C. Multi-ROC curve analysis demonstrated that the nomogram provided an accurate prediction ability with $\mathrm{AUC}=0.769$ for 1-year OS and 0.776 for 3-year OS (Fig. 9D-E), which was higher than the AUC value of the risk score and other clinical factors. DCA was used to compare the net benefit of the nomogram model with grade and clinical stage in patients with $\mathrm{GC}$, and the results demonstrated that the nomogram exhibited superior clinical practicality than the traditional clinical parameters (tumor grade and clinical stage) in predicting the prognosis of patients with GC (Fig. 9F).

\section{Gene Set Enrichment Analysis}

GSEA was conducted to evaluate the potential biological mechanism of the ferroptosis-related IncRNA prognostic signature. According to the GSEA results, the high-risk group was positively related to 24 gene sets ( $P$ value $<0.05$ and FDR $<0.25$ ), and was closely relevant to "basal cell carcinoma," "melanoma," "cell adhesion molecules," "cytokine and cytokine receptor interaction," "ECM receptor interaction," "Focal adhesion," "GAP junction," "regulation of actin cytoskeleton," and "TGF- $\beta$ signaling pathway," as shown in Fig. 10. These pathways are mainly tumor-related and immune-regulated pathways.

\section{Immune Cell Infiltration and Immune-Related Pathways}

Lastly, to discuss the immune state between the high- and low-risk groups, we calculated the scores of 16 immune cells and 13 immune function-related pathways in patients with GC based on SSGSEA analysis and then compared the difference between the two groups. The results revealed that the infiltration level of immune cells, including B cells, DCs, iDCs, macrophages, mast cells, neutrophils, NK cells, pDCs, and Treg cells were significantly upregulated in the high-risk groups $(P<0.05)($ Fig. $11 \mathrm{~A})$. Regarding the immune-related pathways, APC co-stimulation, CCR, and type II IFN response were significantly upregulated in the high-risk group $(P<0.05)$ (Fig. 11B).

\section{Discussion}

The roles of IncRNAs in GC have been extensively studied in recent years, with a focus on progression, metastasis, and prognosis. It has been reported that the expression levels of IncRNAs AGAP2-AS1 and HOXA11-AS are significantly correlated with poorer prognosis and shorter OS in patients with GC, and IncRNA HOXA11-AS is tightly correlated with tumor size, TNM stage, and lymph node metastasis of GC $(17,18)$. Previous studies have found that the expression levels of IncRNAs SNHG17 and B3GALT5-AS1 in GC tissues are significantly correlated with increased invasion depth, lymphatic metastasis, and advanced TNM stage $(19,20)$. However, the expression of IncRNAs DGCR5 and ARHGAP27P1 are 
significantly downregulated in GC tissues, and its downregulation was closely associated with increased invasion depth, advanced TNM stage, and lymphatic metastasis $(20,21)$. Moreover, IncRNAs may affect ferroptosis in various ways, and it has been reported that IncRNA GABPB1-AS1 in HCC leads to the downregulation of the gene encoding Peroxiredoxin-5 (PRDX5) peroxidase, suppression of cellular antioxidant capacity, eventual induction of ferroptosis, and high GABPB1-AS1 levels in patients with HCC, which correlated with improved OS (22). Wu et al. (23) revealed that IncRNA NEAT1 regulates ferroptosis and ferroptosis sensitivity in non-small-cell lung cancer by targeting acyl-CoA synthetase long-chain family member 4 (ACSL4), which may regulate ferroptosis sensitivity. Seven ferroptosis-related IncRNA signatures were constructed in colon adenocarcinoma (COAD), and the prognostic value of these IncRNAs was confirmed in patients with COAD (24). Nevertheless, ferroptosis-related IncRNAs in GC have not been previously studied.

In this study, we focused on the prognostic value of ferroptosis-related IncRNA signatures. In the primary cohort, we first identified 16 prognostic ferroptosis-related IncRNAs based on the TCGA dataset using univariate Cox regression analysis and log-rank test. We then constructed a prognostic model with eight ferroptosis-related IncRNAs using a multivariate Cox regression method. Kaplan-Meier analysis showed that the survival of patients with GC in the high-risk group was worse than that of patients in the low-risk group. The eight ferroptosis-related IncRNA signatures, especially age, sex, tumor grade, and clinical stage, were also relevant to poor OS in different subgroups of patients with GC. We further carried out univariate and multivariate Cox regression analyses and ROC curve analysis. The results demonstrated that the signature provided more sensitivity and specificity and acted as an independent indicator for patients with GC. Next, we established a nomogram based on age, sex, grade, clinical stage, and risk score, and the calibration curve, multi-ROC curve, and DCA were used to verify the accuracy and practicability of the nomogram. All the verified results indicated that the nomogram showed a satisfactory uniformity with actual survival and better clinical practicality than the traditional methods. In addition, internal verification showed that the risk score model could accurately predict OS in patients with GC.

Among the eight prognostic ferroptosis-related IncRNAs (AL365181.3, MIR3142HG, PVT1, LINC01315, AL353804.1, HAGLR, AC005586.1, and AC245041.1) found in GC, PVT1 was significantly upregulated in gastric adenocarcinoma (GAC) compared to matched adjacent normal tissues. Its expression level was positively correlated with larger tumor size, deeper wall invasion, lymph node metastases, and short survival duration, indicating that PVT1 is a poor prognosticator as well as a therapeutic target in GAC (25). It is also a poor prognosticator of esophageal adenocarcinoma (EAC) as its expression was upregulated in EAC compared to paired Barrett's esophagus and normal esophageal tissues. Higher expression of PVT1 was closely associated with poor differentiation, lymph node metastases, and shorter survival (26). LINC01315 was found to be poorly expressed in oral squamous cell carcinoma (OSCC), and LINC01315 knockdown enhanced OSCC cell proliferation, invasion, and migration but dampened their apoptosis by the miR-211 /DLG3 axis (27). Conversely, in colorectal carcinoma (CRC), LINC01315 was significantly upregulated and facilitated the growth and invasive phenotypes of CRC cells by sponging miR-205-3p. Similarly, Ren et al. (28) found that the expression levels of LINC01315 were higher in 
papillary thyroid cancer (PTC) tissues and cell lines than in noncancerous tissues or cells, and it accelerated the growth and invasion of PTC cells by sponging miR-497-5p. HAGLR showed a positive correlation with the rates of lymphatic metastasis and distant metastasis but was negatively correlated with OS in HCC. It was found to be upregulated in HCC tissues compared to para-cancerous tissues (29). Yang et al. reported that HAGLR acts as a microRNA-143-5p sponge to upregulate epithelialmesenchymal transition and metastatic potential in esophageal cancer by regulating LAMP3 (30). One study reported that HAGLR promotes lung adenocarcinoma progression by recruiting DNMT1 to modulate promoter methylation and expression of E2F1 (31). Nevertheless, the other five prognostic ferroptosis-related IncRNAs (AL365181.3, MIR3142HG, AL353804.1, AC005586.1, and AC245041.1) have been rarely reported and are worthy of further research. Moreover, to date, no study has linked ferroptosisassociated IncRNAs to the prognosis of GC. Therefore, the construction of a prognostic ferroptosisassociated IncRNA signature may provide invaluable insights for current prognostic predictions of GC.

Subsequently, we explored the related functions of the eight-IncRNA signature and defined the underlying mechanism in GC. GSEA demonstrated that these IncRNAs were mainly involved in cell adhesion, cancer pathway, and immune function regulation, including "basal cell carcinoma," "melanoma," "cell adhesion molecules," "Focal adhesion," "ECM receptor interaction," "GAP junction, "regulation of actin cytoskeleton, "TGF- $\beta$ signaling pathway," and "cytokine and cytokine receptor interaction." It is well known that adhesion molecules play a vital role in cell recognition, cell activation and signal transduction, cell proliferation and differentiation, and cell extension and movement, which are the molecular basis of important physiological and pathological processes such as immune response, inflammation, coagulation, tumor metastasis, and wound healing. A previous study has demonstrated that the level of intercellular adhesion molecule-1 (sICAM-1) is strongly associated with tumor stage, lymph node metastasis, and tumor metastasis in colorectal cancer (32). The TGF- $\beta$ signaling pathway is closely linked to the occurrence, development, and metastasis of tumors. In the early stage of tumorigenesis, the TGF- $\beta$ signaling pathway can inhibit cell proliferation and block the cell cycle from $\mathrm{G} 1$ to $S$ by inducing the expression of related proteins such as p15, p21, and p27 (33). Nevertheless, TGF- $\beta$ acts as a stimulating molecule in the tumor progression stage, promoting growth, infiltration, and metastasis by inducing immune escape and promoting angiogenesis and epithelial-mesenchymal transformation (34).

The relationship between ferroptosis and tumor immunity has aroused extensive interest in recent years. Ferroptosis is a newly regulated cell death mechanism driven by oxidative injury and promotes lipid peroxidation in an iron-dependent manner. The interaction between ferroptosis and lipid metabolism is critical for tumor immunomodulation. High mobility group box 1 (HMGB1), a biomarker of ferroptosis, was previously reported to promote M1 macrophage polarization through the HMGB1-AGE signaling pathway, and knockdown of HMGB1 decreased derastin-induced ROS generation and cell death via the Ras-JNK/p38 pathway (35). In addition, immune cells regulate tumor ferroptosis; interferon-gamma (IFNY) released from activated CD8 $+T$ cells downregulates the expression of SLC3A2 and SLC7A11, which are two subunits of the glutamate-cystine antiporter system xc-. Consequently, GSH cannot scavenge lipid peroxides through GPX4 and promotes lipid peroxidation and ferroptosis (36). In turn, increased ferroptosis contributes to the anti-tumor efficacy of immunotherapy in tumor cells (36). Given the close 
association between ferroptosis and the tumor immune system, we investigated the roles of immune infiltrating cells and immune function-related pathways in the tumor microenvironment in the prognosis of GC. sSGSEA analysis revealed that immune cells, including B cells, DCs, iDCs, macrophages, mast cells, neutrophils, NK cells, pDCs, and Treg cells, were significantly enriched in the high-risk group, and APC co-stimulation, CCR, and type II IFN response pathways were more activated in high-risk patients.

Although we established a prognostic signature and the nomogram performed well in predicting the survival of patients with GC, the limitations of our study deserve attention. First, this eight ferroptosisrelated IncRNA prognostic signature was constructed and evaluated using limited data and clinical information from the TCGA database and was not verified in external cohorts, which restricted the practicality and generalizability of the prognostic model. Moreover, some of these IncRNAs have rarely been reported and are worthy of further research. It is critical to verify these bioinformatics prediction results with functional experiments and molecular mechanism studies of the eight ferroptosis-related IncRNAs.

\section{Conclusion}

We constructed and identified a novel ferroptosis-related prognostic risk model comprising eight IncRNAs (AL365181.3, MIR3142HG, PVT1, LINC01315, AL353804.1, HAGLR, AC005586.1, and AC245041.1) in GC, and then built a predicted nomogram. The risk model signature and nomogram may improve prognostic predictive accuracy and guide individualized treatment for patients with GC.

\section{Materials And Methods}

\section{Data Acquisition and Processing}

The high-throughput sequencing (HTSeq) of fragments per kilobase of transcript per million mapped reads (FPKM) of GC tissues, including 375 tumor samples and 32 normal control samples, were downloaded from TCGA (https://portal.gdc.cancer.gov/). After eliminating the data of genes with low expression levels with an average value $<0.5$, the data included information of 930 IncRNAs and 12952 protein-coding genes according to the ENSEMBL database (http://asia.ensembl.org/index.html). After removing the data of normal samples and patients with less than 30 days follow-up time, the remaining data of 334 patients with GC were assigned as the primary cohort, and 172 of these were assigned randomly as the validation cohort. The data of ferroptosis-related genes were obtained from FerrDb (37), which is a database that collects information on iron death-related markers, regulatory factors, and iron death-related diseases. In total, 259 ferroptosis-related genes were identified and classified as 111 marker genes, 108 driver genes, and 69 suppressor genes.

\section{Screening Ferroptosis-Related LncRNA in GC}

Among the data of the 259 ferroptosis-related genes downloaded from FerrDb, the data of 217 autophagy-related gene matrix in GC were extracted by using the "limma" package of R 4.0.4 software 
(http:///www.r-project.org/). Pearson correlation coefficient analysis was performed to identify ferroptosis-related IncRNAs according to the criteria of | correlation coefficient $\mid>0.3$, and $p<0.001$. In total, 741 ferroptosis-related IncRNAs were screened for further verification.

\section{Establishment and Identification of Ferroptosis-Related LncRNA Prognostic Model for GC}

To extract the prognostic ferroptosis-related IncRNAs, we performed univariate Cox regression analyses and log-rank tests. The prognostic results included a hazard ratio (HR), 95\% confidence interval (Cl), and log-rank test $P$ value. Subsequently, we incorporated prognostic ferroptosis-related IncRNA candidates into a multivariate Cox regression analysis to screen for independent prognostic signatures. Next, we established an optimum prognostic risk model based on the lowest Akaike information criterion (AIC = 1213) by using "Survival" R package. The correlation between the expression of each identified prognostic ferroptosis-related IncRNA and survival was evaluated using the Kaplan-Meier survival analysis and log-rank test; a $p$ value $<0.05$ was considered statistically significant. Thereafter, according to the multivariate Cox regression coefficient and expression value of each IncRNA in patients with GC, the risk score of each patient was evaluated using the risk score equation as follows:

risk score $=$ coef $($ IncRNA1 $) \times \exp ($ IncRNA1 $)+\operatorname{coef}($ IncRNA2 $) \times \exp ($ IncRNA2 $)+\ldots+$ coef $($ IncRNAn $) \times \exp$ (IncRNAn).

where coef (IncRNA) represents the regression coefficient and exp (IncRNA) is the expressive value of each ferroptosis-related IncRNA. The median risk score was calculated and used to divide the patients into high- and low-risk groups. Kaplan-Meier survival analysis of the primary and validation cohorts was performed to assess the overall survival (OS) difference between the low- and high-risk groups. Meanwhile, stratification analysis was performed based on the following clinicopathological features: age ( $\leq 65$ and $>65$ years), sex (male and female), grade (grade $1-2$ and grade 3 ), and clinical stage (stage I-II and stage III-IV); $p<0.05$ was considered statistically significant. A time-dependent Receiver Operating Characteristic (time-ROC) curve was constructed to evaluate the predictive accuracy of the prognostic model for 1-, 2-, and 3-year OS via "survivalROC" R package.

Subsequently, univariate and multivariate Cox regression analyses were performed to explore whether the risk score model could be used as an independent factor for the prognosis of patients with GC, integrating the following clinicopathological factors: age, sex, grade, clinical stage, and TNM stage. The final prognostic results were presented with a $\mathrm{HR}, 95 \% \mathrm{Cl}$, and a $\mathrm{p}$ value $<0.05$ which was considered statistically significant. A multi-indicator ROC (multi-ROC) curve was drawn to assess the predictive accuracy of risk score and other clinicopathological features for survival via "survivalROC" R package; the area under the curve (AUC) of ROC curve estimated the sensitivity and specificity of prediction factors.

The relevance between prognostic ferroptosis-related IncRNAs and their co-expressed mRNAs was analyzed using "ggalluvial" R package of the Cytoscape 3.8.2 software (http://www.cytos cape.org/).

\section{Construction and Validation of Nomogram}


Based on the risk score and clinical characteristics (including age, sex, grade, and clinical stage), we established a nomogram to predict 1- and 3-year survival probability of patients with GC by using "rms" $\mathrm{R}$ packages. We then performed internal verification to assess the predictive precision of the nomogram. A calibration curve was drawn to appraise the uniformity of the predicted results. A multi-ROC curve was sketched to assess the predictive accuracy of the nomogram model. Decision curve analysis (DCA) was used to estimate the net benefit of the prognostic nomogram in patients with $\mathrm{GC}$, where the abscissa represented the threshold probability and ordinates represented the net income.

\section{Gene Set Enrichment Analysis}

We performed Gene Set Enrichment Analysis (GSEA; version 4.1.0) to identify the differential genes and pathways between the high- and low-risk groups and further analyze the potential biological mechanisms of the ferroptosis-related IncRNA prognostic signature. The c2.cp.kegg.v7.4.symbols.gmt downloaded from the Molecular Signatures Database (http://www.gsea-msigdb.org/gsea/msigdb/collections.jsp) was used, and a false discovery rate (FDR q-value) $<0.05$ was set to identify the significantly enriched pathways.

\section{Immune Cell Infiltration and Immune-related Pathways}

According to the ferroptosis-related IncRNA prognostic model, single-sample GSEA (sSGSEA) was conducted to quantitatively analyze the difference in infiltrating level of immune cells and immunerelated pathways between high- and low-risk groups in patients with GC by using "gsva" and "GSEABase" $\mathrm{R}$ package (38). The result was visualized using "ggpubr" $\mathrm{R}$ package of boxplot. Statistical significance was set at $p<0.05$.

\section{Statistical Analysis}

Statistical analyses were performed using R software (https://www.r-project.org/, version 4.0.4) and SPSS version 24 (IBM, New York, USA). The chi-square test was used to analyze the baseline characteristics of patients with hepatocellular carcinoma (HCC). Cox regression analysis was used to build a prognostic model. First, univariate Cox analysis and log-rank tests were conducted to screen for prognostic EMT-related IncRNA candidates with $p<0.05$. Then, we used a multivariate Cox model to reduce data dimensionality and calculate the risk score. Kaplan-Meier analysis was used to evaluate the survival difference between the two risk groups. A time-ROC curve was used to estimate the predictive abilities of the risk models. The prognostic value of the nomogram for 1- and 3-year OS was assessed using the calibration curve, multi-ROC curve, and DCA. All statistical tests were two-sided, and statistical significance was set at $p<0.05$.

\section{Abbreviations}

GC

gastric cancer; LncRNA:Iong non-coding RNA; TCGA:The Cancer Genome Atlas; HR:hazard ratio; OS:overall survival; ROC:receiver operating characteristic; multi-ROC:multi-indicator receiver operating 
characteristic; AUC:area under the curve; DCA:decision curve analysis; GSEA:gene set enrichment analysis; SsGSEA:single-sample gene set enrichment analysis; Cl:Confidence Interval; HR:Hazard Ratio.

\section{Declarations}

\section{Conflict of Interest}

The authors declare no competing interests.

\section{Ethical approval statement}

The study was approved by the ethics committee of the Second Affiliated Hospital of Nanchang University.

\section{Consent for publication}

Not applicable.

\section{$4 \quad$ Author Contributions}

JB W conceived and designed the study. SL $H$ and $D L$ were responsible for the collection and analysis of the research information. SL H was involved in drafting the manuscript. LL Z, LY S, and JB W critically and carefully revised the manuscript. All the authors read and approved the final manuscript.

\section{$5 \quad$ Funding}

This work was supported by the National Natural Science Foundation of China (No. 82060435).

\section{Acknowledgments}

We acknowledge and appreciate our colleagues for their valuable efforts and comments on this paper.

\section{Data Availability Statement}

The datasets presented in this study can be found in online repositories. The names of the repository/repositories and accession number(s) can be found in the article/Supplementary Material.

\section{References}

1. Mao R, Wang Z, Zhang Y, Chen Y, Liu Q, Zhang T, et al. Development and validation of a novel prognostic signature in gastric adenocarcinoma. Aging. 2020;12(21):22233-52.

2. Joshi SS, Badgwell BD. Current treatment and recent progress in gastric cancer. CA Cancer J Clin. 2021. 
3. Duraes C, Almeida GM, Seruca R, Oliveira C, Carneiro F. Biomarkers for gastric cancer: prognostic, predictive or targets of therapy? Virchows Arch. 2014;464(3):367-78.

4. Yuan L, Xu ZY, Ruan SM, Mo S, Qin JJ, Cheng XD. Long non-coding RNAs towards precision medicine in gastric cancer: early diagnosis, treatment, and drug resistance. Mol Cancer. 2020;19(1):96.

5. Tirada N, Aujero M, Khorjekar G, Richards S, Chopra J, Dromi S, et al. Breast Cancer Tissue Markers, Genomic Profiling, and Other Prognostic Factors: A Primer for Radiologists. Radiographics. 2018;38(7):1902-20.

6. Dixon SJ, Lemberg KM, Lamprecht MR, Skouta R, Zaitsev EM, Gleason CE, et al. Ferroptosis: an irondependent form of nonapoptotic cell death. Cell. 2012;149(5):1060-72.

7. Yang WS, Kim KJ, Gaschler MM, Patel M, Shchepinov MS, Stockwell BR. Peroxidation of polyunsaturated fatty acids by lipoxygenases drives ferroptosis. Proc Natl Acad Sci U S A. 2016;113(34):E4966-75.

8. Shi Z, Zhang L, Zheng J, Sun H, Shao C. Ferroptosis: Biochemistry and Biology in Cancers. Front Oncol. 2021;11:579286.

9. Liu Z, Zhao Q, Zuo ZX, Yuan SQ, Yu K, Zhang Q, et al. Systematic Analysis of the Aberrances and Functional Implications of Ferroptosis in Cancer. iScience. 2020;23(7):101302.

10. Jiang X, Yan Q, Xie L, Xu S, Jiang K, Huang J, et al. Construction and Validation of a FerroptosisRelated Prognostic Model for Gastric Cancer. J Oncol. 2021;2021:6635526.

11. Deng X, Bi Q, Chen S, Chen X, Li S, Zhong Z, et al. Identification of a Five-Autophagy-Related-IncRNA Signature as a Novel Prognostic Biomarker for Hepatocellular Carcinoma. Front Mol Biosci. 2020;7:611626.

12. Necula L, Matei L, Dragu D, Neagu Al, Mambet C, Nedeianu S, et al. Recent advances in gastric cancer early diagnosis. World J Gastroenterol. 2019;25(17):2029-44.

13. Fan Y, Wang YF, Su HF, Fang N, Zou C, Li WF, et al. Decreased expression of the long noncoding RNA LINC00261 indicate poor prognosis in gastric cancer and suppress gastric cancer metastasis by affecting the epithelial-mesenchymal transition. J Hematol Oncol. 2016;9(1):57.

14. Wang M, Mao C, Ouyang L, Liu Y, Lai W, Liu N, et al. Long noncoding RNA LINC00336 inhibits ferroptosis in lung cancer by functioning as a competing endogenous RNA. Cell Death Differ. 2019;26(11):2329-43.

15. Mao C, Wang X, Liu Y, Wang M, Yan B, Jiang Y, et al. A G3BP1-Interacting IncRNA Promotes Ferroptosis and Apoptosis in Cancer via Nuclear Sequestration of p53. Cancer Res. 2018;78(13):3484-96.

16. Tang Y, Li C, Zhang YJ, Wu ZH. Ferroptosis-Related Long Non-Coding RNA signature predicts the prognosis of Head and neck squamous cell carcinoma. Int J Biol Sci. 2021;17(3):702-11.

17. Liu Z, Chen Z, Fan R, Jiang B, Chen X, Chen Q, et al. Over-expressed long noncoding RNA HOXA11-AS promotes cell cycle progression and metastasis in gastric cancer. Mol Cancer. 2017;16(1):82. 
18. Qi F, Liu X, Wu H, Yu X, Wei C, Huang X, et al. Long noncoding AGAP2-AS1 is activated by SP1 and promotes cell proliferation and invasion in gastric cancer. J Hematol Oncol. 2017;10(1):48.

19. Feng W, Zong W, Li Y, Shen X, Cui X, Ju S. Abnormally expressed long noncoding RNA B3GALT5-AS1 may serve as a biomarker for the diagnostic and prognostic of gastric cancer. J Cell Biochem. 2020;121(1):557-65.

20. Zhang G, Xu Y, Wang S, Gong Z, Zou C, Zhang H, et al. LncRNA SNHG17 promotes gastric cancer progression by epigenetically silencing of p15 and p57. J Cell Physiol. 2019;234(4):5163-74.

21. Zhang G, Xu Y, Zou C, Tang Y, Lu J, Gong Z, et al. Long noncoding RNA ARHGAP27P1 inhibits gastric cancer cell proliferation and cell cycle progression through epigenetically regulating p15 and p16. Aging. 2019;11(20):9090-110.

22. Qi W, Li Z, Xia L, Dai J, Zhang Q, Wu C, et al. LncRNA GABPB1-AS1 and GABPB1 regulate oxidative stress during erastin-induced ferroptosis in HepG2 hepatocellular carcinoma cells. Sci Rep. 2019;9(1):16185.

23. Wu H, Liu A. Long non-coding RNA NEAT1 regulates ferroptosis sensitivity in non-small-cell lung cancer. J Int Med Res. 2021;49(3):300060521996183.

24. Cai HJ, Zhuang ZC, Wu Y, Zhang YY, Liu X, Zhuang JF, et al. Development and validation of a ferroptosis-related IncRNAs prognosis signature in colon cancer. Bosn J Basic Med Sci. 2021.

25. Li Y, Song S, Pizzi MP, Han G, Scott AW, Jin J, et al. LncRNA PVT1 Is a Poor Prognosticator and Can Be Targeted by PVT1 Antisense Oligos in Gastric Adenocarcinoma. Cancers (Basel). 2020;12(10).

26. Xu Y, Li Y, Jin J, Han G, Sun C, Pizzi MP, et al. LncRNA PVT1 up-regulation is a poor prognosticator and serves as a therapeutic target in esophageal adenocarcinoma. Mol Cancer. 2019;18(1):141.

27. Chen FB, Wu P, Zhou R, Yang QX, Zhang X, Wang RR, et al. LINC01315 Impairs microRNA-211Dependent DLG3 Downregulation to Inhibit the Development of Oral Squamous Cell Carcinoma. Front Oncol. 2020;10:556084.

28. Ren J, Zhang FJ, Wang JH, Tang JD. LINC01315 promotes the aggressive phenotypes of papillary thyroid cancer cells by sponging miR-497-5p. Kaohsiung J Med Sci. 2021.

29. Li YH, Huang GM, Wang W, Zang HL. LncRNA HAGLR exacerbates hepatocellular carcinoma through negatively regulating miR-6785-5p. Eur Rev Med Pharmacol Sci. 2020;24(18):9353-60.

30. Yang C, Shen S, Zheng X, Ye K, Sun Y, Lu Y, et al. Long noncoding RNA HAGLR acts as a microRNA143-5p sponge to regulate epithelial-mesenchymal transition and metastatic potential in esophageal cancer by regulating LAMP3. Faseb j. 2019;33(9):10490-504.

31. Guo X, Chen Z, Zhao L, Cheng D, Song W, Zhang X. Long non-coding RNA-HAGLR suppressed tumor growth of lung adenocarcinoma through epigenetically silencing E2F1. Exp Cell Res. 2019;382(1):111461.

32. Kang X, Wang F, Xie JD, Cao J, Xian PZ. Clinical evaluation of serum concentrations of intercellular adhesion molecule-1 in patients with colorectal cancer. World J Gastroenterol. 2005;11(27):4250-3.

33. Meulmeester E, Ten Dijke P. The dynamic roles of TGF- $\beta$ in cancer. J Pathol. 2011;223(2):205-18. 
34. Kim BG, Malek E, Choi SH, Ignatz-Hoover JJ, Driscoll JJ. Novel therapies emerging in oncology to target the TGF- $\beta$ pathway. J Hematol Oncol. 2021;14(1):55.

35. Ye F, Chai W, Xie M, Yang M, Yu Y, Cao L, et al. HMGB1 regulates erastin-induced ferroptosis via RASJNK/p38 signaling in HL-60/NRAS(Q61L) cells. Am J Cancer Res. 2019;9(4):730-9.

36. Wang W, Green M, Choi JE, Gijón M, Kennedy PD, Johnson JK, et al. CD8(+) T cells regulate tumour ferroptosis during cancer immunotherapy. Nature. 2019;569(7755):270-4.

37. Zhou N, Bao J. FerrDb: a manually curated resource for regulators and markers of ferroptosis and ferroptosis-disease associations. Database (Oxford). 2020;2020.

38. Rooney MS, Shukla SA, Wu CJ, Getz G, Hacohen N. Molecular and genetic properties of tumors associated with local immune cytolytic activity. Cell. 2015;160(1-2):48-61.

\section{Figures}

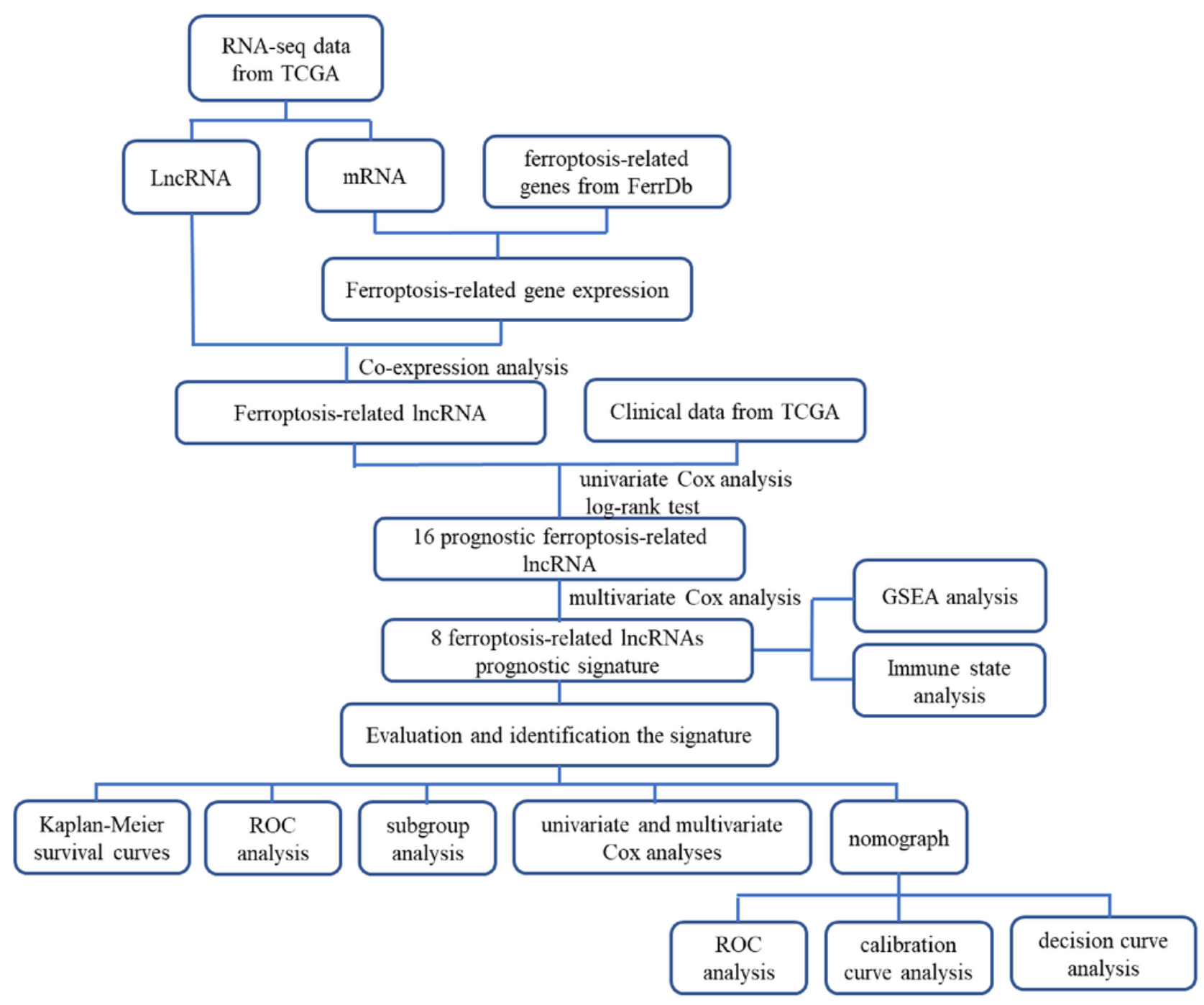

Figure 1 
Flowchart of the analysis. LncRNA: long noncoding RNA; TCGA: The Cancer Genome Atlas; ROC: Receiver Operating Characteristic; GSEA: Gene set enrichment analysis.

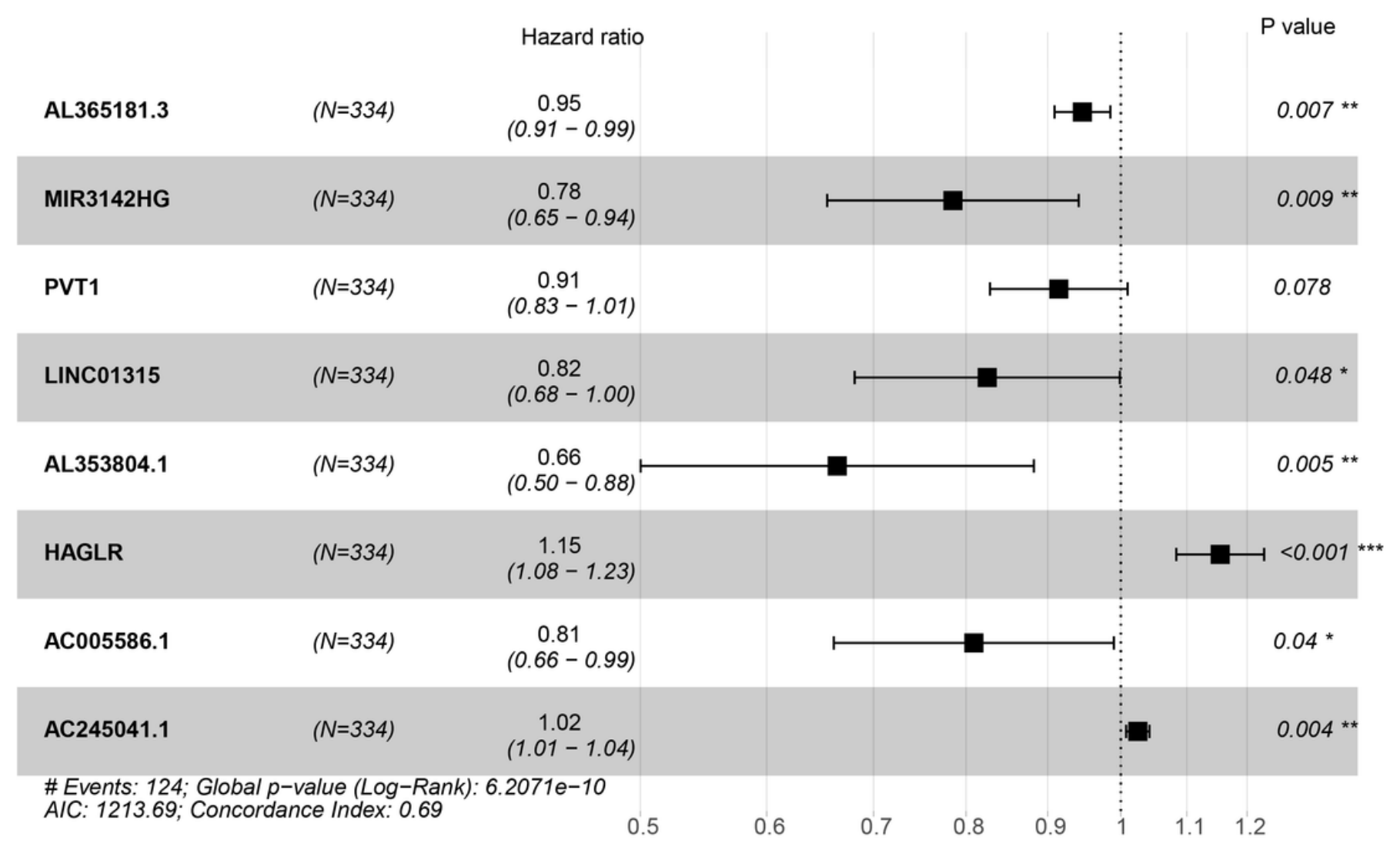

\section{Figure 2}

Construction of ferroptosis-related IncRNAs with independent prognosis in gastric cancer. The forest plot showed the Hazard Ratio (95\% Confidence Interval) and $p$ value of IncRNAs by multivariate Cox proportional hazards analysis. ${ }^{\star} \mathrm{P}<0.05 ;{ }^{\star \star} \mathrm{P}<0.01 ; \star \star \star \mathrm{P}<0.001$. 
A

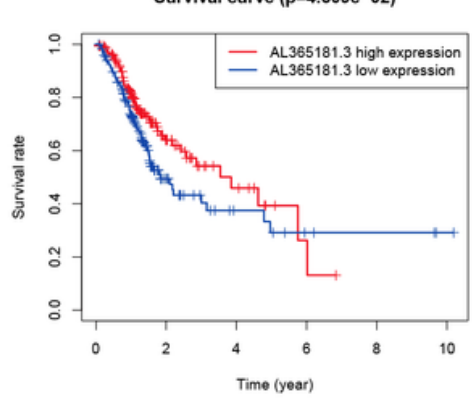

E

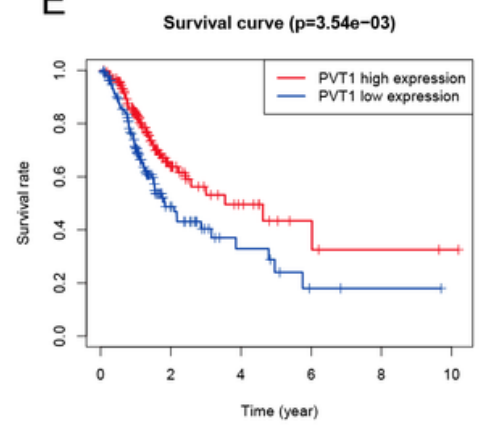

B

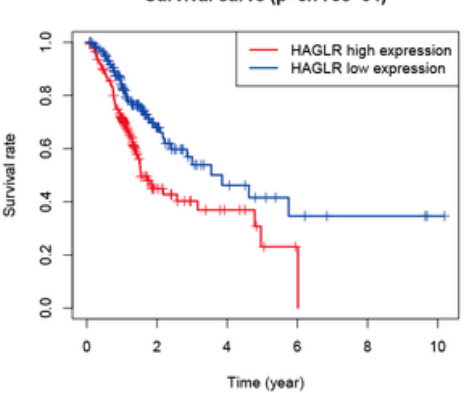

$\mathrm{F}$

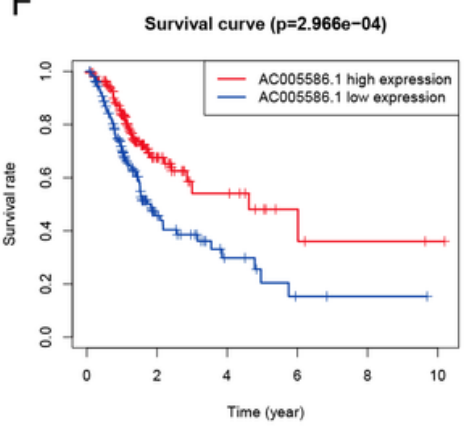

C

Survival curve $(p=2.406 e-02)$

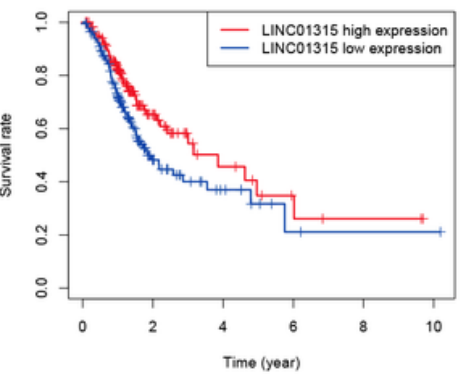

G

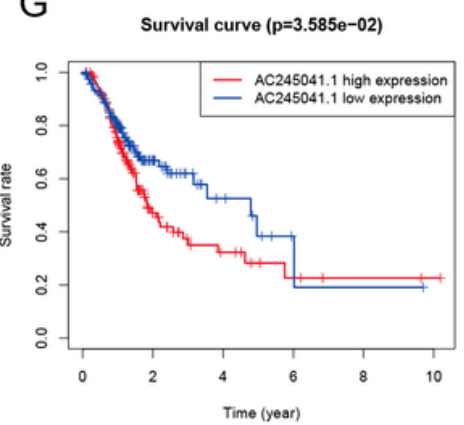

D Survival curve $\left(p=3.122 e^{-02)}\right.$

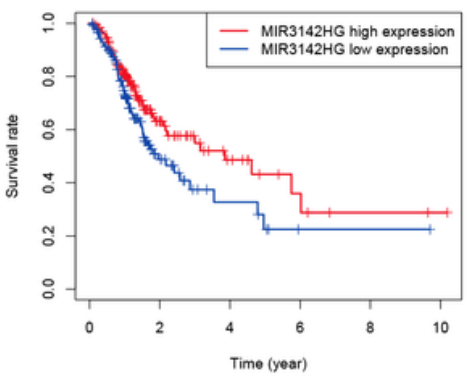

$\mathrm{H}$ Survival curve (p=1.238e-03)

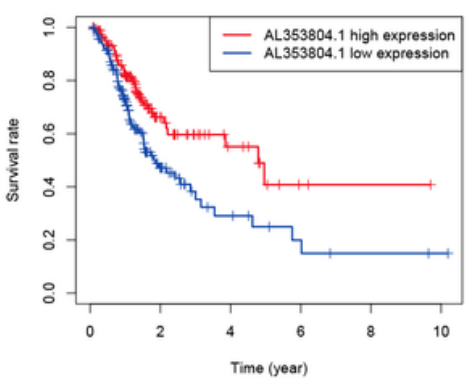

Figure 3

Kaplan-Meier survival analysis of the association between the eight ferroptosis-related IncRNAs and overall survival of patients with gastric cancer $(A-H)$. 
A

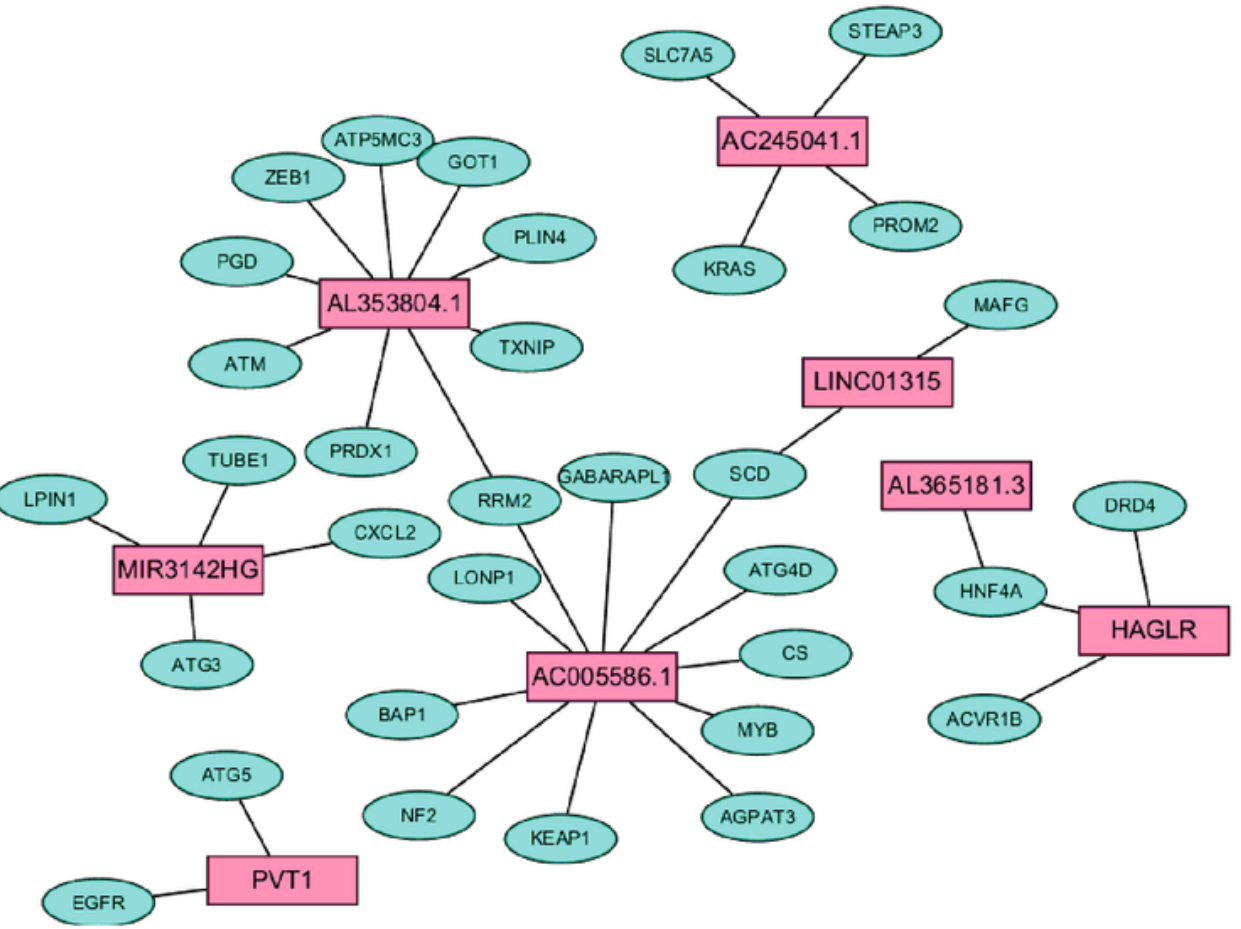

B

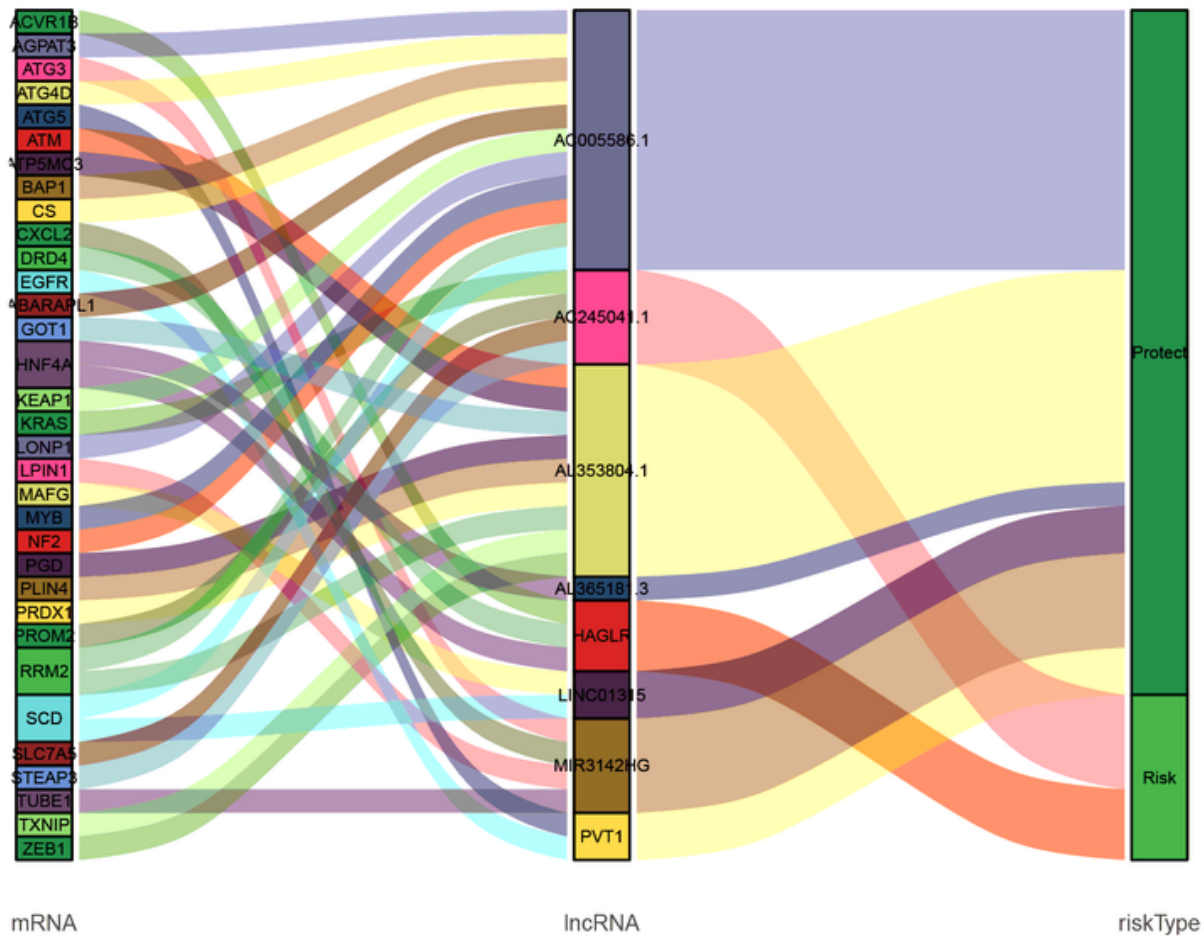

Figure 4

Co-expression network of the prognostic ferroptosis-related IncRNAs-mRNAs visualized using Cytoscape(A) and Sankey diagram(B) 


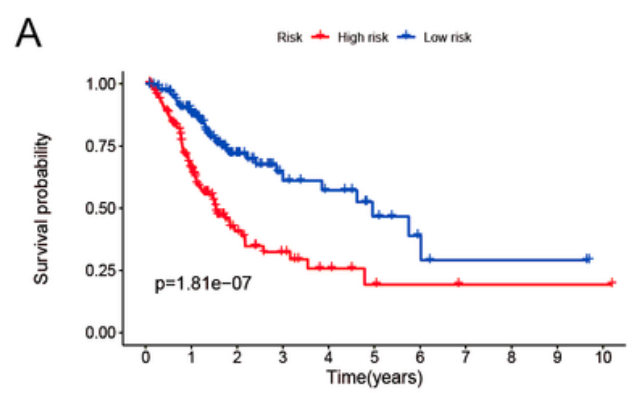

C
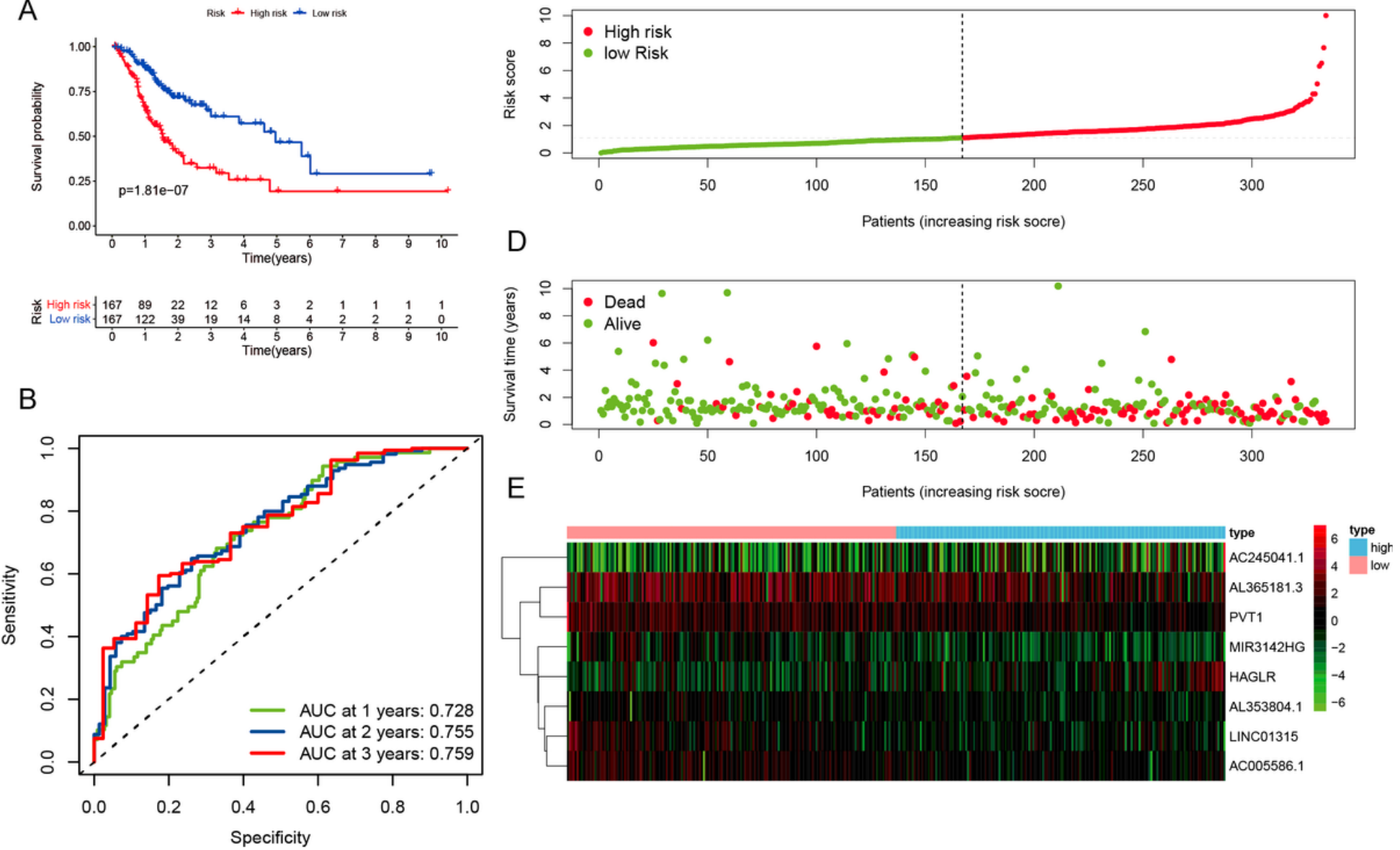

Figure 5

Prognosis analysis of the eight ferroptosis-related IncRNAs signature in The Cancer Genome Atlas (TCGA) primary cohort (A-E). (A): Kaplan-Meier survival analysis of the high- and low-risk groups. (B): Time-dependent receiver operating characteristic (time-ROC) curve evaluated the precision of this prognostic model in 1-, 2-, and 3-year OS. (C). The risk score curves based on the risk score of each patient. (D): The scatter plots based on the survival status of each patient; the green dots represent living and red dots represent dead patients. (E): The heatmap displayed the expression levels of the eight ferroptosis-related IncRNAs in the high- and low-risk groups. 
A

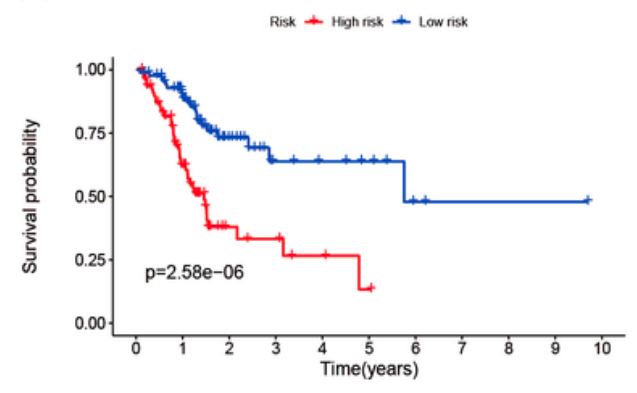

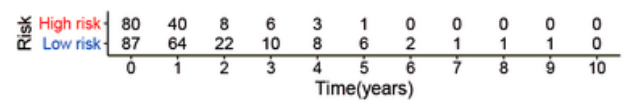

B

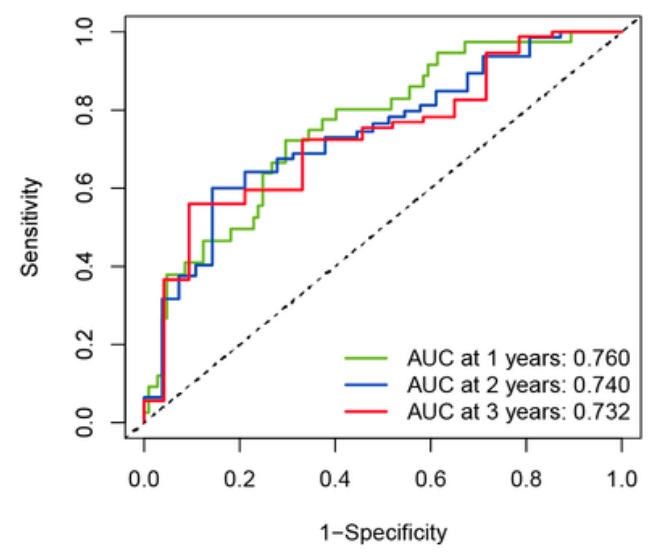

C
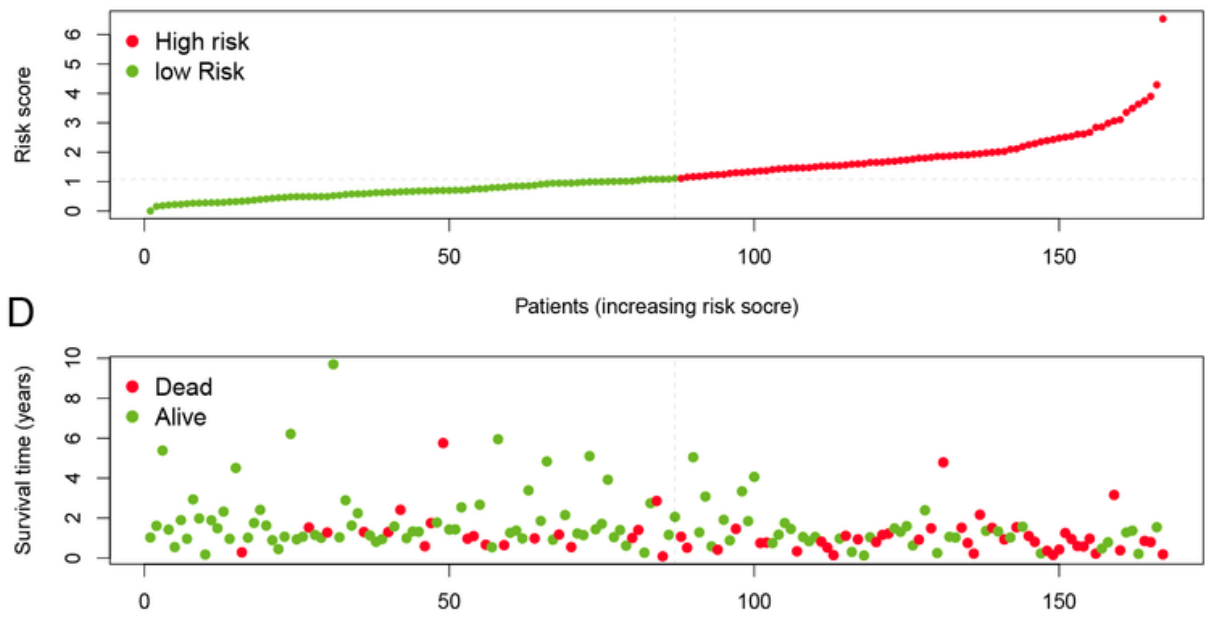

E

Patients (increasing risk socre)

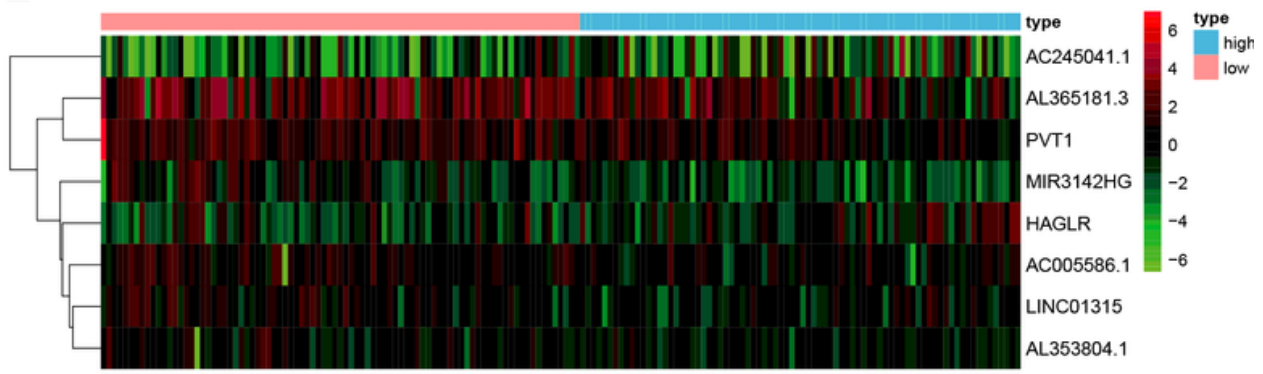

\section{Figure 6}

Prognosis analysis of the eight ferroptosis-related IncRNAs signature in The Cancer Genome Atlas (TCGA) validation cohort (A-E). (A): Kaplan-Meier survival analysis of the high- and low-risk groups. (B): Time-dependent receiver operating characteristic (time-ROC) curve evaluated the precision of this prognostic model in 1-, 2-, and 3-year OS. (C): The risk score curves based on the risk score of each patient. (D): The scatter plots based on the survival status of each patient. The green dots represent living and red dots represent dead patients. (E): The heatmap displayed the expression levels of the eight ferroptosis-related IncRNAs in the high- and low-risk groups. 

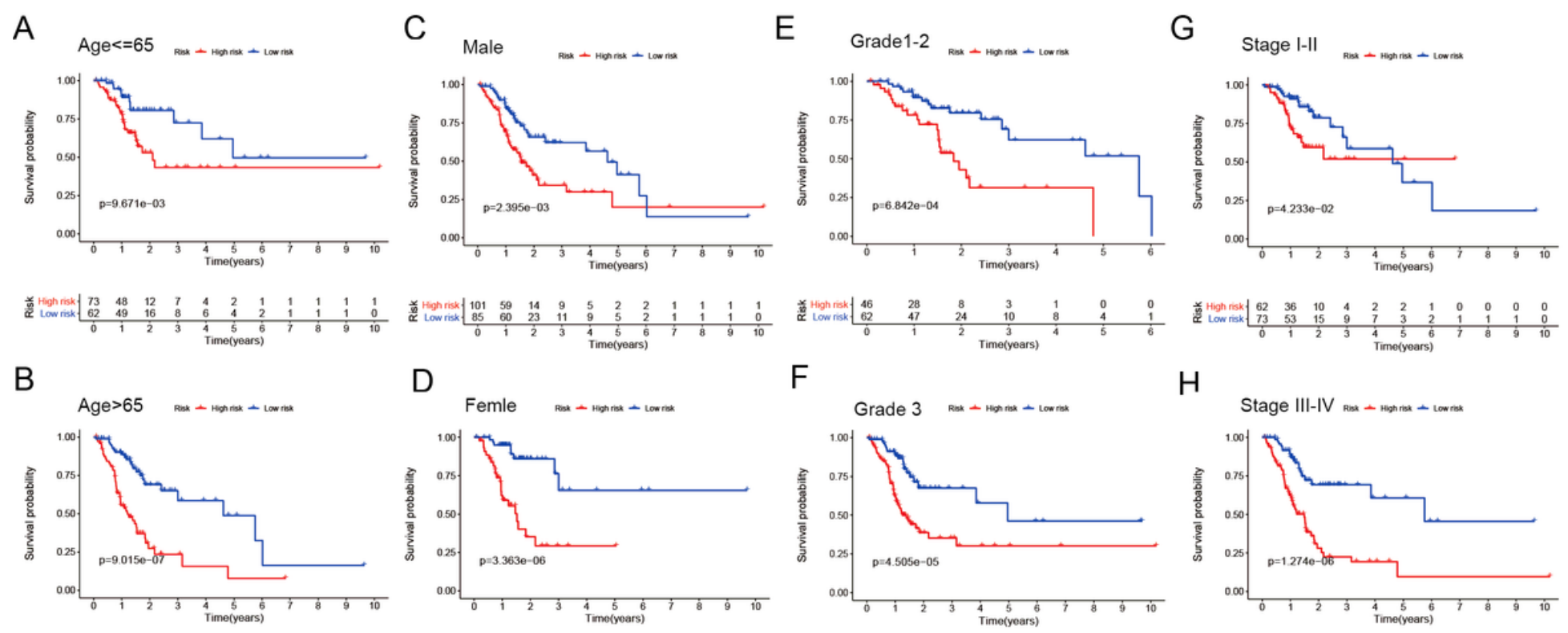

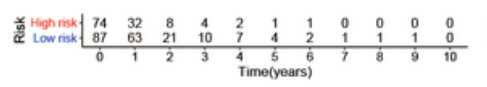
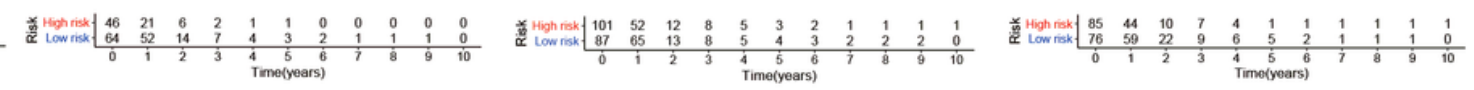

\section{Figure 7}

Stratification analysis of the correlation between the risk groups and survival based on age, gender, grade, and clinical stage (A-H). (A): Age $\leq 65 ;(B)$ : Age > 65; (C): Male; (D): Female; (E): Grade 1-2; (F): Grade 3;

(G): Stage I-II; (H): Stage III-IV. 
primary cohort

A

\begin{tabular}{|c|c|}
\hline & Hazard ratio \\
\hline ge & $0.0241 .022(1.003-1.042)$ \\
\hline ender & $0.0720 .679(0.445-1.035)$ \\
\hline ade & $0.1101 .357(0.934-1.972)$ \\
\hline age & $<0.0011 .478(1.172-1.863)$ \\
\hline T & $0.0391 .289(1.013-1.641)$ \\
\hline & $0.1181 .728(0.871-3.429)$ \\
\hline & $0.0111 .252(1.053-1.490)$ \\
\hline
\end{tabular}

C

$\begin{array}{lrr} & \text { pvalue } & \text { Hazard ratio } \\ \text { age } & 0.002 & 1.033(1.012-1.054) \\ \text { gender } & 0.109 & 0.701(0.455-1.083) \\ \text { grade } & 0.136 & 1.349(0.910-2.000) \\ \text { stage } & 0.194 & 1.342(0.861-2.091) \\ \mathrm{T} & 0.471 & 1.130(0.810-1.577) \\ \mathrm{M} & 0.322 & 1.550(0.651-3.688) \\ \mathrm{N} & 0.715 & 1.049(0.813-1.352) \\ \text { riskScore } & <0.0011 .097(1.061-1.133)\end{array}$

E

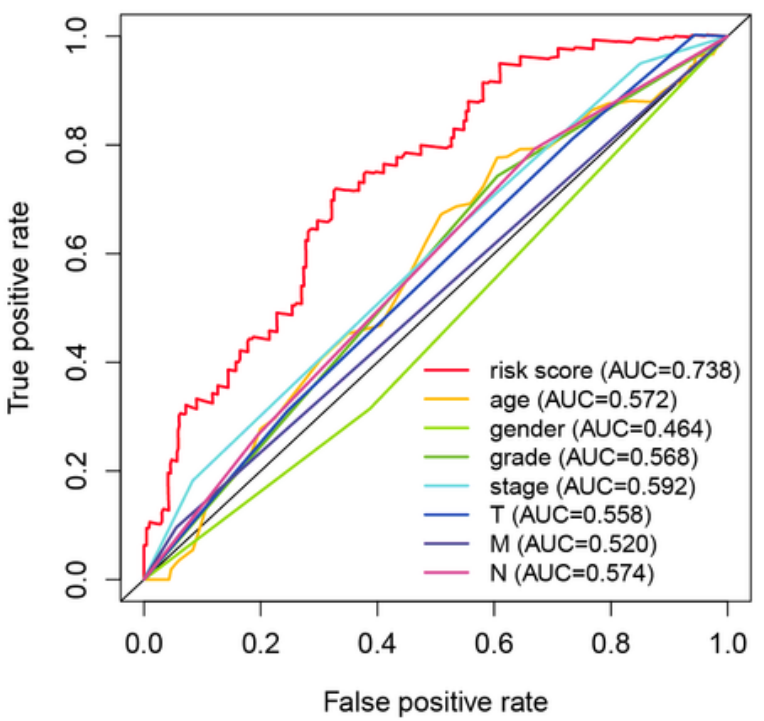

B
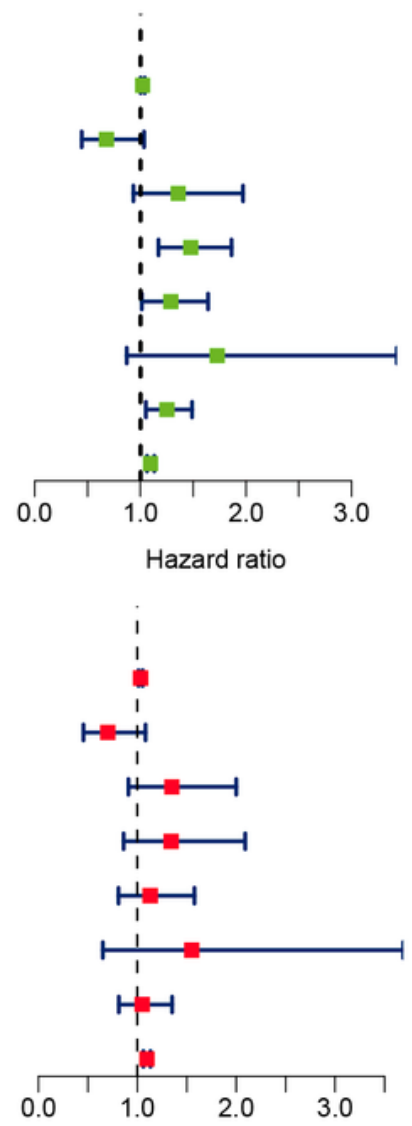

Hazard ratio

F

\section{validation cohort}
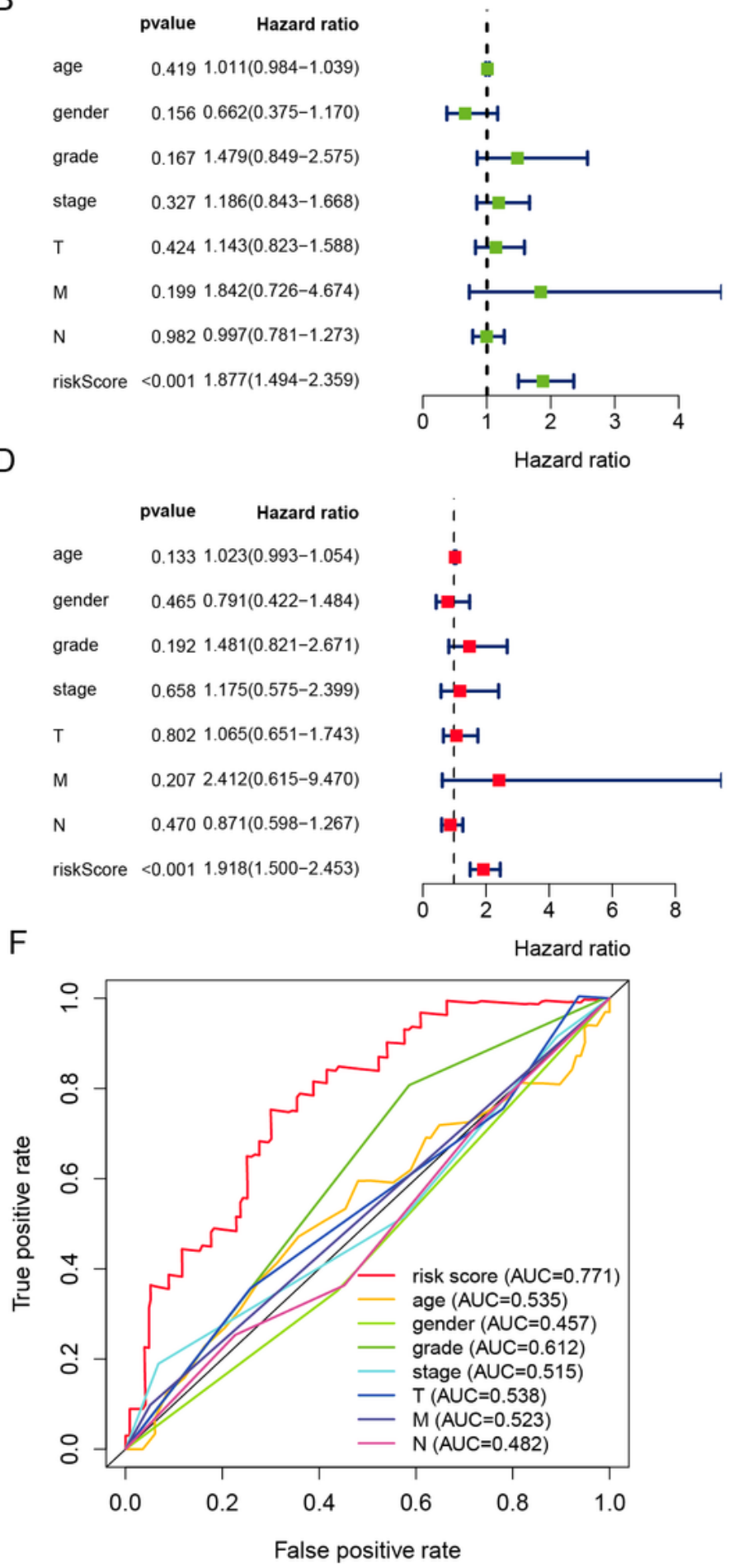

\section{Figure 8}

Prognostic value of risk score and clinicopathological features in primary and validation cohorts (A-F). (A): Univariate Cox regression analysis in primary cohort. (B): Univariate Cox regression analysis in validation cohort. (C): Multivariate Cox regression analysis in primary cohort. (D): Multivariate Cox regression analysis in validation cohort. (E) Multi-indicator receiver operating characteristic (muti-ROC) 
curve analysis in primary cohort. (F) Multi-indicator receiver operating characteristic (muti-ROC) curve analysis in validation cohort.

A

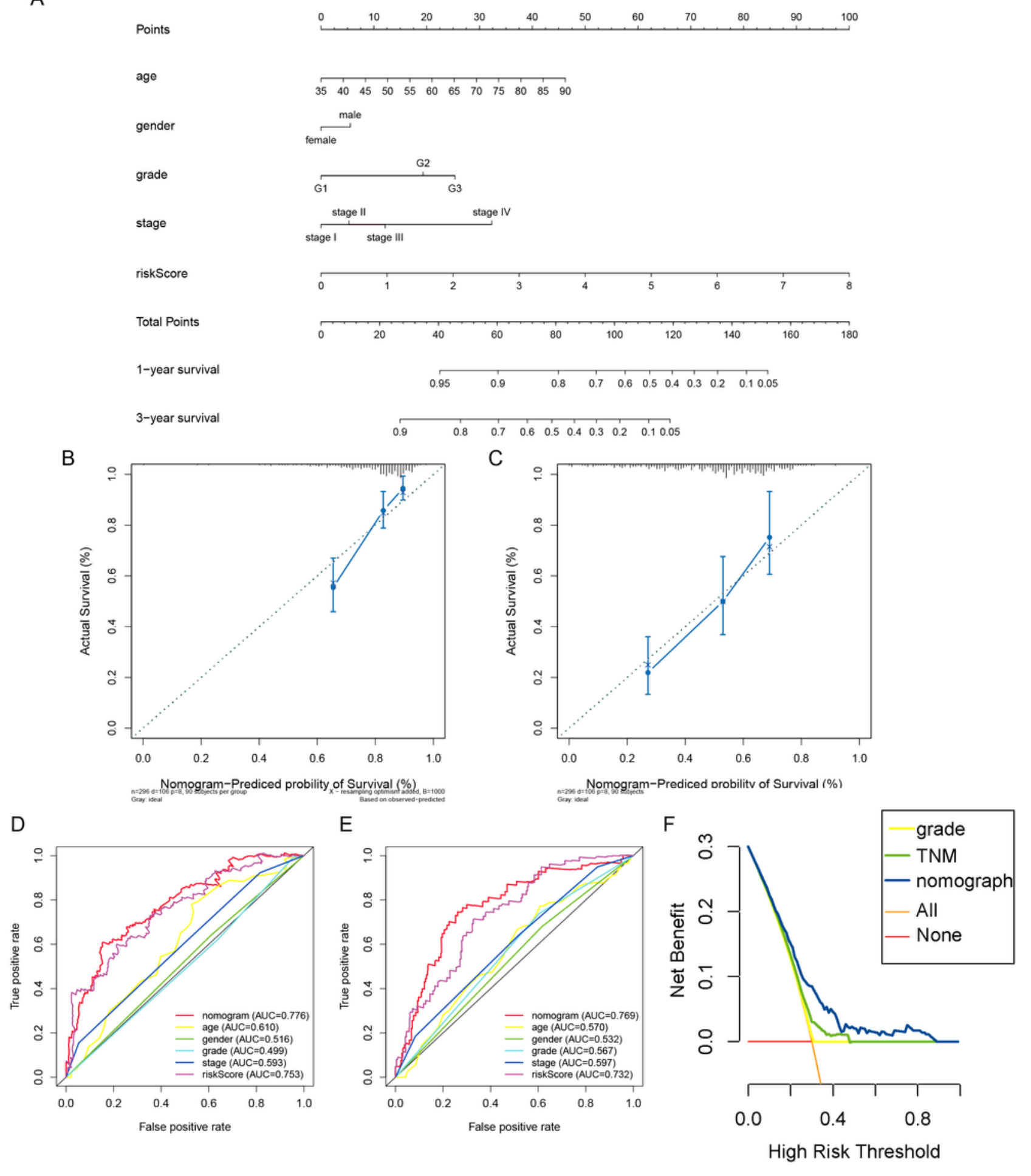

Figure 9

Construction and verification of the predictive nomogram for patients with gastric cancer from The Cancer Genome Atlas (TCGA) (A-F). (A) Nomogram predicting the probability of 1- and 3-year OS for the prognosis of patients with gastric cancer. $(B, C)$ Calibration curve for the predictive accuracy of the 
nomogram. The $\mathrm{Y}$-axis represents actual survival, and the $\mathrm{X}$-axis represents nomogram-predicted survival. (D, E) The receiver operating characteristic (ROC) curve analysis of the nomogram for 1- and 3-year OS. (F) Decision curve analysis evaluating the clinical practicality of the nomogram model.
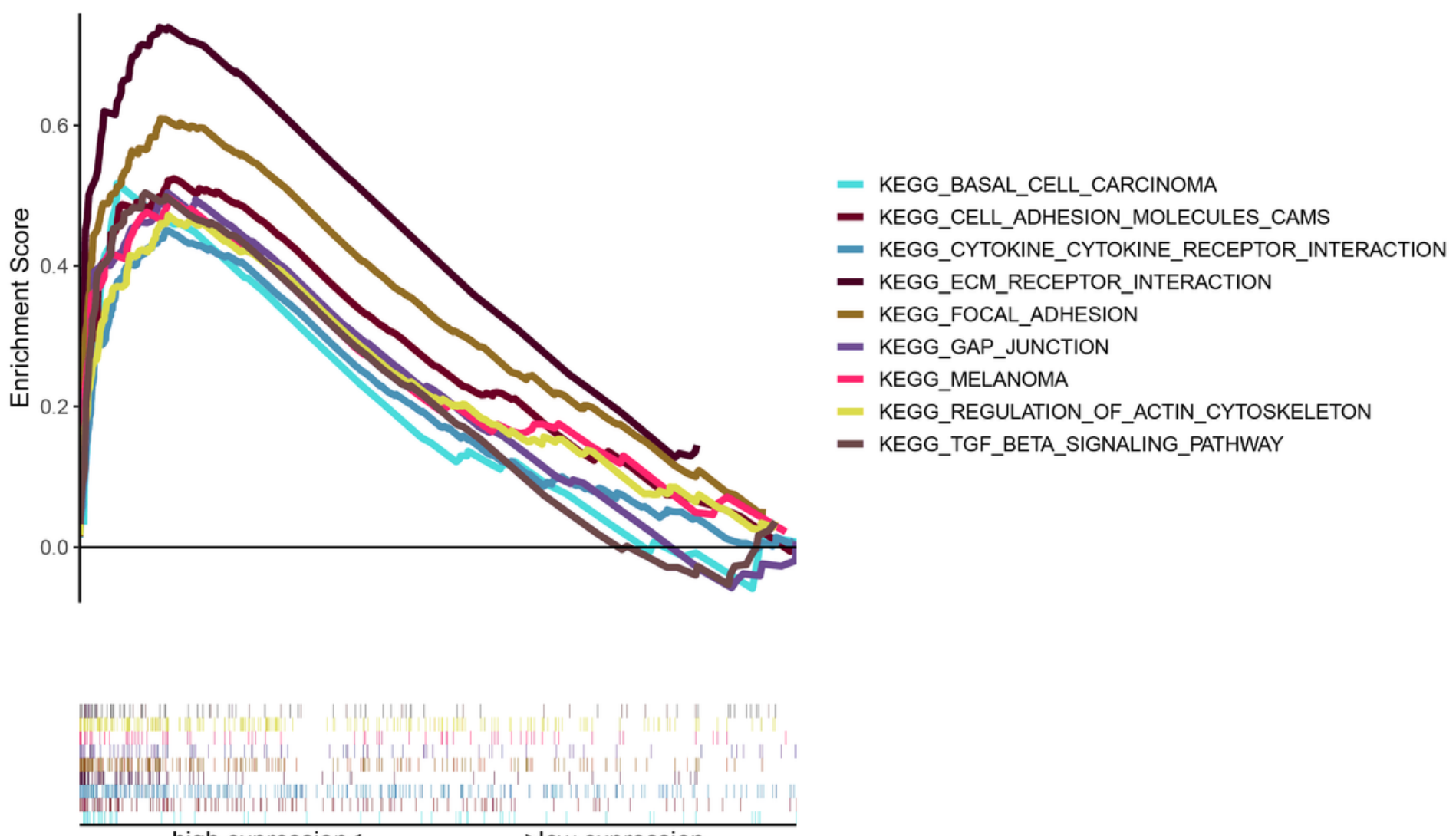

high expression<------------>low expression

\section{Figure 10}

Gene set enrichment analysis (GSEA) of low- and high-risk group patients with gastric cancer

A Risk 追 low 追 high

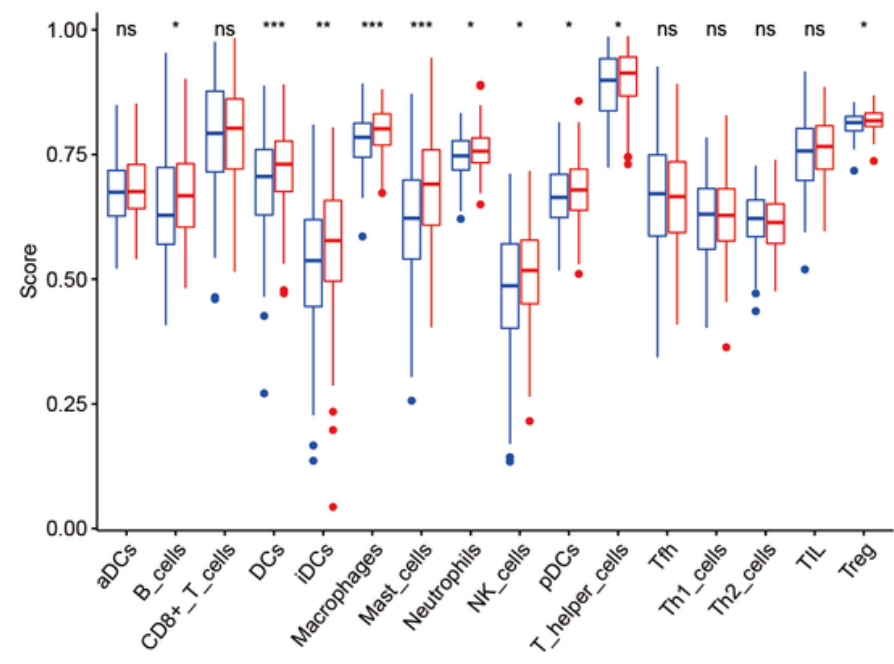

B

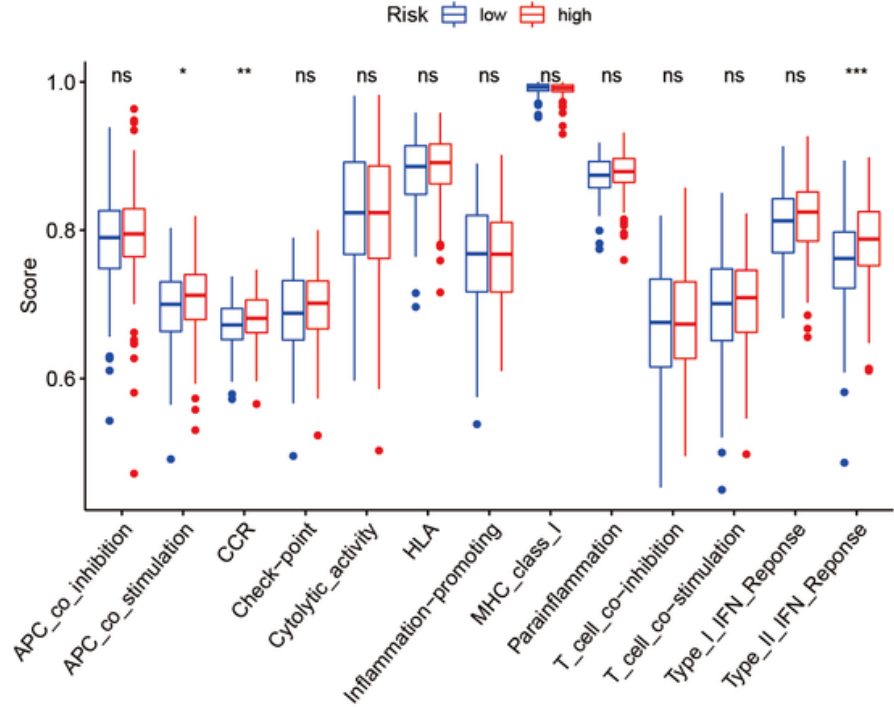


Immune state analysis of the prognostic ferroptosis-related signature based on single-sample gene set enrichment analysis (sSGSEA) (A-B). (A) Comparison of the infiltration of 16 immune cells between lowand high-risk groups. (B) Comparison of the immune functions between low- and high-risk groups. ns: no statistical significance; ${ }^{*} \mathrm{P}<0.05 ; * \star \mathrm{P}<0.01 ; * \star \star \mathrm{P}<0.001$. 